Acta Crystallographica Section E

Structure Reports

Online

ISSN 1600-5368

\section{Rajenahally S. Narasegowda, Hemmige S. Yathirajan, ${ }^{a}$ Padmarajaiah Nagaraja ${ }^{a}$ and Daniel E. Lynch ${ }^{\text {b* }}$}

${ }^{a}$ Department of Studies in Chemistry, University of Mysore, Manasagangotri, Mysore 570 006, India, and ${ }^{\mathbf{b}}$ School of Science and the Environment, Coventry University, Coventry CV1 5FB, England

Correspondence e-mail: apx106@coventry.ac.uk

\author{
Key indicators \\ Single-crystal X-ray study \\ $T=120 \mathrm{~K}$ \\ Mean $\sigma(\mathrm{C}-\mathrm{C})=0.002 \AA$ \\ $R$ factor $=0.028$ \\ $w R$ factor $=0.078$ \\ Data-to-parameter ratio $=18.3$
}

For details of how these key indicators were automatically derived from the article, see http://journals.iucr.org/e.

\title{
4-(2-Chloroethyl)morpholinium chloride
}

The structure of the title compound, $\mathrm{C}_{6} \mathrm{H}_{13} \mathrm{ClNO}^{+} \cdot \mathrm{Cl}^{-}$, comprises a cation with the morpholine ring in the chair conformation, and a single hydrogen-bonding association between the morpholinium NH group and the $\mathrm{Cl}^{-}$anion.

\section{Comment}

The title compound, (I), is used as an intermediate for the synthesis of the antispasmodic drug pinaverium bromide, and is also used as an intermediate for the synthesis of biologically active heterocycles (Baronnet et al., 1974). A search of the Cambridge Structural Database (Version 5.26; Allen, 2002) reveals that there are 90 known structures that contain the morpholinium cation. Of these there are 24 that have an $N$ ethyl chain, or longer, including the structure of 4-(2-fluoroethyl)morpholinium chloride (Briggs et al., 2004). This compound crystallizes in monoclinic space group $P 2_{1} / n$, with the morpholine ring in the chair conformation and a single hydrogen-bonding association between the morpholinium $\mathrm{NH}$ group and the $\mathrm{Cl}^{-}$anion $(\mathrm{N} \cdots \mathrm{Cl}=3.036 \AA)$.

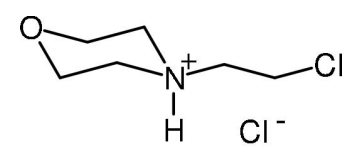

(I)

The structure of the title compound comprises a cation with the morpholine ring also in the chair conformation (Fig. 1), and a single hydrogen-bonding association similarly between

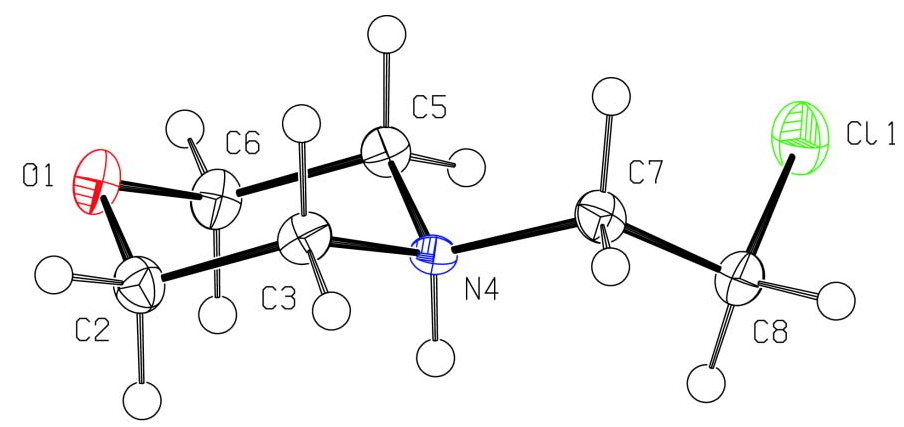

Figure 1

The molecular configuration and atom-numbering scheme for (I). Displacement ellipsoids are drawn at the $50 \%$ probability level and $\mathrm{H}$ atoms are drawn as spheres of arbitrary radius.
Received 22 February 2005 Accepted 25 February 2005 Online 4 March 2005 
the morpholinium NH group and the $\mathrm{Cl}^{-}$anion (Table 1). Three torsion angles that define the conformation of the chloroethyl chain are $\mathrm{C} 2-\mathrm{C} 3-\mathrm{N} 4-\mathrm{C} 7\left[-177.50(12)^{\circ}\right], \mathrm{C} 3-$ $\mathrm{N} 4-\mathrm{C} 7-\mathrm{C} 8 \quad\left[162.71(13)^{\circ}\right]$ and $\mathrm{N} 4-\mathrm{C} 7-\mathrm{C} 8-\mathrm{Cl} 1$ $\left[86.66(15)^{\circ}\right]$. The equivalent angles in the fluoro analogue are $178.46,-78.85$ and $-173.68^{\circ}$, respectively.

\section{Experimental}

An equimolar mixture of morpholine $(0.87 \mathrm{~g}, 10 \mathrm{mmol})$, anhydrous $\mathrm{K}_{2} \mathrm{CO}_{3}(1.38 \mathrm{~g}, 10 \mathrm{mmol})$ and 1-bromo-2-chloroethane $(1.43 \mathrm{~g}$, $10 \mathrm{mmol}$ ) was stirred at room temperature in dimethylformamide $(10 \mathrm{ml})$ for $6 \mathrm{~h}$. The collected product was subsequently converted to the hydrochloride salt using isopropyl alcohol and $\mathrm{HCl}$ (80:20). Crystals of compound (I) were grown from methanol.

$$
\begin{aligned}
& \text { Crystal data } \\
& \mathrm{C}_{6} \mathrm{H}_{13} \mathrm{ClNO}^{+} \cdot \mathrm{Cl}^{-} \\
& M_{r}=186.07 \\
& \text { Triclinic, } P \overline{1} \\
& a=6.9876(3) \AA \\
& b=8.1549(4) \AA \\
& c=8.6495(3) \AA \\
& \alpha=63.530(2)^{\circ} \\
& \beta=85.004(3)^{\circ} \\
& \gamma=85.179(2)^{\circ} \\
& V=438.97(3) \AA^{\circ}
\end{aligned}
$$$$
Z=2
$$$$
D_{x}=1.408 \mathrm{Mg} \mathrm{m}^{-3}
$$$$
\text { Mo } K \alpha \text { radiation }
$$$$
\text { Cell parameters from } 1914
$$$$
\text { reflections }
$$$$
\theta=2.9-27.5^{\circ}
$$$$
\mu=0.68 \mathrm{~mm}^{-1}
$$$$
T=120(2) \mathrm{K}
$$$$
\text { Plate, colourless }
$$$$
0.28 \times 0.24 \times 0.06 \mathrm{~mm}
$$

\section{Data collection}

Nonius KappaCCD diffractometer $\varphi$ and $\omega$ scans

Absorption correction: multi-scan (SADABS; Sheldrick, 2003)

$T_{\min }=0.833, T_{\max }=0.961$

7581 measured reflections

1716 independent reflections

\section{Refinement}

Refinement on $F^{2}$

$R\left[F^{2}>2 \sigma\left(F^{2}\right)\right]=0.028$

$w R\left(F^{2}\right)=0.078$

$S=0.94$

1716 reflections

94 parameters

$\mathrm{H}$ atoms treated by a mixture of independent and constrained refinement

1494 reflections with $I>2 \sigma(I)$

$R_{\text {int }}=0.032$

$\theta_{\max }=26.0^{\circ}$

$h=-8 \rightarrow 8$

$k=-10 \rightarrow 9$

$l=-10 \rightarrow 10$

$$
\begin{aligned}
& w=1 /\left[\sigma^{2}\left(F_{o}{ }^{2}\right)+(0.0469 P)^{2}\right. \\
& +0.2143 P] \\
& \text { where } P=\left(F_{o}^{2}+2 F_{c}^{2}\right) / 3 \\
& (\Delta / \sigma)_{\max }<0.001
\end{aligned}
$$

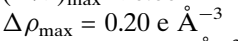

$$
\begin{aligned}
& \Delta \rho_{\min }=-0.28{\mathrm{e} \AA^{-3}}^{-3}
\end{aligned}
$$

Table 1

Hydrogen-bonding geometry $\left(\AA{ }^{\circ}{ }^{\circ}\right)$.

\begin{tabular}{lllll}
\hline$D-\mathrm{H} \cdots A$ & $D-\mathrm{H}$ & $\mathrm{H} \cdots A$ & $D \cdots A$ & $D-\mathrm{H} \cdots A$ \\
\hline $\mathrm{N} 4-\mathrm{H} 4 \cdots \mathrm{Cl} 2$ & $0.883(19)$ & $2.16(2)$ & $3.0435(14)$ & $178(2)$ \\
\hline
\end{tabular}

The $\mathrm{H}$ atom attached to the $\mathrm{N}$ atom was located in a difference Fourier synthesis and its positional parameters were refined. Other $\mathrm{H}$ atoms were included in the refinement at calculated positions, in the riding-model approximation, with a $\mathrm{C}-\mathrm{H}$ distance of $0.99 \AA$. The isotropic displacement parameters for all $\mathrm{H}$ atoms were set equal to $1.25 U_{\text {eq }}$ of the carrier atom.

Data collection: COLLECT (Hooft, 1998); cell refinement: DENZO (Otwinowski \& Minor, 1997) and COLLECT; data reduction: DENZO and COLLECT; program(s) used to solve structure: SHELXS97 (Sheldrick, 1997); program(s) used to refine structure: SHELXL97 (Sheldrick, 1997); molecular graphics: PLATON (Spek, 2003); software used to prepare material for publication: SHELXL97.

The authors thank the EPSRC National Crystallography Service (Southampton, England) and acknowledge the use of the EPSRC's Chemical Database Service at Daresbury (Fletcher et al., 1996).

\section{References}

Allen, F. H. (2002). Acta Cryst. B58, 380-388.

Baronnet, R., Foussard, B. O., Bretaudeau, J. \& Hubert, F. (1974). Eur. J. Med. Chem. 9, 182-187.

Briggs, C. R. S., Allen, M. J., O’Hagan, D., Tozer, D. J., Slawin, A. M. Z., Goeta, A. E. \& Howard, J. A. K. (2004). Org. Biomol. Chem. 2, 732-740.

Fletcher, D. A., McMeeking, R. F. \& Parkin, D. J. (1996). J. Chem. Inf. Comput. Sci. 36, 746-749.

Hooft, R. W. W. (1998). COLLECT. Nonius BV, Delft, The Netherlands.

Otwinowski, Z. \& Minor, W. (1997). Methods in Enzymology, Vol. 276, Macromolecular Crystallography, Part A, edited by C. W. Carter Jr and R. M. Sweet, pp. 307-326. New York: Academic Press.

Sheldrick, G. M. (1997). SHELXS97 and SHELXL97. University of Göttingen, Germany.

Sheldrick, G. M. (2003). SADABS. Version 2.10. Bruker AXS Inc., Madison, Wisconsin, USA.

Spek, A. L. (2003). J. Appl. Cryst. 36, 7-13. 


\section{supporting information}

Acta Cryst. (2005). E61, o849-o850 [https://doi.org/10.1107/S1600536805006136]

\section{4-(2-Chloroethyl)morpholinium chloride}

Rajenahally S. Narasegowda, Hemmige S. Yathirajan, Padmarajaiah Nagaraja and Daniel E. Lynch

4-(2-Chloroethyl)morpholinium chloride

Crystal data

$\mathrm{C}_{6} \mathrm{H}_{13} \mathrm{ClNO}^{+} \cdot \mathrm{Cl}^{-}$

$M_{r}=186.07$

Triclinic, $P \overline{1}$

Hall symbol: -P 1

$a=6.9876(3) \AA$

$b=8.1549(4) \AA$

$c=8.6495(3) \AA$

$\alpha=63.530(2)^{\circ}$

$\beta=85.004(3)^{\circ}$

$\gamma=85.179(2)^{\circ}$

$V=438.97(3) \AA^{3}$

\section{Data collection}

Nonius KappaCCD diffractometer

Radiation source: Bruker Nonius FR591 rotating anode

$10 \mathrm{~cm}$ confocal mirrors monochromator

Detector resolution: 9.091 pixels $\mathrm{mm}^{-1}$

$\varphi$ and $\omega$ scans

Absorption correction: multi-scan

(SADABS; Sheldrick, 2003)

Refinement

Refinement on $F^{2}$

Least-squares matrix: full

$R\left[F^{2}>2 \sigma\left(F^{2}\right)\right]=0.028$

$w R\left(F^{2}\right)=0.078$

$S=0.94$

1716 reflections

94 parameters

0 restraints

Primary atom site location: structure-invariant direct methods
$Z=2$

$F(000)=196$

$D_{\mathrm{x}}=1.408 \mathrm{Mg} \mathrm{m}^{-3}$

Melting point: $458 \mathrm{~K}$

Mo $K \alpha$ radiation, $\lambda=0.71073 \AA$

Cell parameters from 1914 reflections

$\theta=2.9-27.5^{\circ}$

$\mu=0.68 \mathrm{~mm}^{-1}$

$T=120 \mathrm{~K}$

Plate, colourless

$0.28 \times 0.24 \times 0.06 \mathrm{~mm}$

$T_{\min }=0.833, T_{\max }=0.961$

7581 measured reflections

1716 independent reflections

1494 reflections with $I>2 \sigma(I)$

$R_{\text {int }}=0.032$

$\theta_{\text {max }}=26.0^{\circ}, \theta_{\min }=3.8^{\circ}$

$h=-8 \rightarrow 8$

$k=-10 \rightarrow 9$

$l=-10 \rightarrow 10$

Secondary atom site location: difference Fourier map

Hydrogen site location: inferred from neighbouring sites

$\mathrm{H}$ atoms treated by a mixture of independent and constrained refinement

$w=1 /\left[\sigma^{2}\left(F_{0}^{2}\right)+(0.0469 P)^{2}+0.2143 P\right]$ where $P=\left(F_{\mathrm{o}}^{2}+2 F_{\mathrm{c}}{ }^{2}\right) / 3$

$(\Delta / \sigma)_{\max }<0.001$

$\Delta \rho_{\max }=0.20 \mathrm{e} \AA^{-3}$

$\Delta \rho_{\min }=-0.28 \mathrm{e} \AA^{-3}$ 
Fractional atomic coordinates and isotropic or equivalent isotropic displacement parameters $\left(\hat{A}^{2}\right)$

\begin{tabular}{|c|c|c|c|c|}
\hline & $x$ & $y$ & $z$ & $U_{\text {iso }} * / U_{\text {eq }}$ \\
\hline O1 & $0.31450(17)$ & $-0.10081(15)$ & $0.17258(14)$ & $0.0211(3)$ \\
\hline $\mathrm{C} 2$ & $0.2513(2)$ & $0.0634(2)$ & $0.0296(2)$ & $0.0206(4)$ \\
\hline $\mathrm{H} 21$ & 0.3005 & 0.0597 & -0.0797 & $0.026^{*}$ \\
\hline $\mathrm{H} 22$ & 0.1090 & 0.0712 & 0.0319 & $0.026^{*}$ \\
\hline $\mathrm{C} 3$ & $0.3210(2)$ & $0.2316(2)$ & $0.0350(2)$ & $0.0171(3)$ \\
\hline H31 & 0.2755 & 0.3437 & -0.0652 & $0.021 *$ \\
\hline H32 & 0.4634 & 0.2269 & 0.0287 & $0.021 *$ \\
\hline N4 & $0.24469(19)$ & $0.23719(18)$ & $0.20020(17)$ & $0.0133(3)$ \\
\hline $\mathrm{H} 4$ & $0.118(3)$ & $0.243(2)$ & $0.203(2)$ & $0.017^{*}$ \\
\hline $\mathrm{C} 5$ & $0.3049(2)$ & $0.0621(2)$ & $0.3499(2)$ & $0.0159(3)$ \\
\hline H51 & 0.4467 & 0.0529 & 0.3546 & $0.020 *$ \\
\hline H52 & 0.2479 & 0.0611 & 0.4592 & $0.020 *$ \\
\hline C6 & $0.2391(2)$ & $-0.0999(2)$ & $0.3312(2)$ & $0.0193(4)$ \\
\hline H61 & 0.0968 & -0.0946 & 0.3346 & $0.024 *$ \\
\hline H62 & 0.2820 & -0.2151 & 0.4295 & $0.024 *$ \\
\hline $\mathrm{C} 7$ & $0.3033(2)$ & $0.4064(2)$ & $0.2071(2)$ & $0.0176(3)$ \\
\hline H71 & 0.4406 & 0.3899 & 0.2337 & $0.022 *$ \\
\hline H72 & 0.2906 & 0.5116 & 0.0917 & $0.022 *$ \\
\hline $\mathrm{C} 8$ & $0.1861(3)$ & $0.4513(2)$ & $0.3400(2)$ & $0.0222(4)$ \\
\hline H81 & 0.1832 & 0.5855 & 0.3010 & $0.028 *$ \\
\hline H82 & 0.0522 & 0.4168 & 0.3460 & $0.028 *$ \\
\hline $\mathrm{Cl1}$ & $0.27566(7)$ & $0.33778(6)$ & $0.55327(6)$ & $0.02923(15)$ \\
\hline $\mathrm{Cl} 2$ & $-0.19260(5)$ & $0.24738(5)$ & $0.21272(5)$ & $0.02018(14)$ \\
\hline
\end{tabular}

Atomic displacement parameters $\left(\AA^{2}\right)$

\begin{tabular}{lllllll}
\hline & $U^{11}$ & $U^{22}$ & $U^{33}$ & $U^{12}$ & $U^{13}$ & $U^{23}$ \\
\hline O1 & $0.0271(7)$ & $0.0174(6)$ & $0.0196(6)$ & $-0.0017(5)$ & $0.0038(5)$ & $-0.0097(5)$ \\
C2 & $0.0225(9)$ & $0.0226(9)$ & $0.0188(8)$ & $-0.0015(7)$ & $-0.0008(7)$ & $-0.0110(7)$ \\
C3 & $0.0183(8)$ & $0.0189(8)$ & $0.0115(7)$ & $-0.0003(6)$ & $0.0018(6)$ & $-0.0048(6)$ \\
N4 & $0.0105(6)$ & $0.0147(7)$ & $0.0139(6)$ & $-0.0015(5)$ & $-0.0008(5)$ & $-0.0054(5)$ \\
C5 & $0.0188(8)$ & $0.0135(8)$ & $0.0130(8)$ & $-0.0014(6)$ & $-0.0011(6)$ & $-0.0036(6)$ \\
C6 & $0.0227(9)$ & $0.0157(8)$ & $0.0179(8)$ & $-0.0043(7)$ & $0.0039(7)$ & $-0.0063(7)$ \\
C7 & $0.0195(8)$ & $0.0124(8)$ & $0.0185(8)$ & $-0.0042(6)$ & $-0.0010(6)$ & $-0.0042(7)$ \\
C8 & $0.0217(9)$ & $0.0188(9)$ & $0.0291(9)$ & $0.0020(7)$ & $-0.0040(7)$ & $-0.0133(8)$ \\
C11 & $0.0381(3)$ & $0.0306(3)$ & $0.0224(2)$ & $-0.0013(2)$ & $-0.00025(19)$ & $-0.0151(2)$ \\
C12 & $0.0122(2)$ & $0.0228(2)$ & $0.0189(2)$ & $-0.00099(16)$ & $-0.00073(15)$ & $-0.00332(18)$ \\
& & & & & &
\end{tabular}

Geometric parameters $\left(\AA,{ }^{\circ}\right)$

\begin{tabular}{llll}
\hline $\mathrm{N} 4-\mathrm{C} 5$ & $1.496(2)$ & $\mathrm{C} 6-\mathrm{C} 5$ & $1.513(2)$ \\
$\mathrm{N} 4-\mathrm{C} 7$ & $1.4995(19)$ & $\mathrm{C} 6-\mathrm{H} 61$ & 0.99 \\
$\mathrm{~N} 4-\mathrm{C} 3$ & $1.4994(19)$ & $\mathrm{C} 6-\mathrm{H} 62$ & 0.99 \\
$\mathrm{~N} 4-\mathrm{H} 4$ & $0.883(19)$ & $\mathrm{C} 5-\mathrm{H} 51$ & 0.99 \\
$\mathrm{C} 2-\mathrm{C} 3$ & $1.515(2)$ & $\mathrm{C} 5-\mathrm{H} 52$ & 0.99
\end{tabular}




\begin{tabular}{|c|c|c|c|}
\hline $\mathrm{C} 3-\mathrm{H} 31$ & 0.99 & $\mathrm{C} 7-\mathrm{C} 8$ & $1.512(2)$ \\
\hline $\mathrm{C} 3-\mathrm{H} 32$ & 0.99 & $\mathrm{C} 7-\mathrm{H} 71$ & 0.99 \\
\hline $\mathrm{C} 2-\mathrm{O} 1$ & $1.427(2)$ & $\mathrm{C} 7-\mathrm{H} 72$ & 0.99 \\
\hline $\mathrm{C} 2-\mathrm{H} 21$ & 0.99 & $\mathrm{C} 8-\mathrm{Cl1}$ & $1.7980(18)$ \\
\hline $\mathrm{C} 2-\mathrm{H} 22$ & 0.99 & $\mathrm{C} 8-\mathrm{H} 81$ & 0.99 \\
\hline $\mathrm{O} 1-\mathrm{C} 6$ & $1.4289(19)$ & $\mathrm{C} 8-\mathrm{H} 82$ & 0.99 \\
\hline $\mathrm{C} 5-\mathrm{N} 4-\mathrm{C} 7$ & $114.05(12)$ & $\mathrm{O} 1-\mathrm{C} 6-\mathrm{H} 62$ & 109.4 \\
\hline $\mathrm{C} 5-\mathrm{N} 4-\mathrm{C} 3$ & $109.14(12)$ & $\mathrm{C} 5-\mathrm{C} 6-\mathrm{H} 62$ & 109.4 \\
\hline $\mathrm{C} 7-\mathrm{N} 4-\mathrm{C} 3$ & $110.77(12)$ & $\mathrm{H} 61-\mathrm{C} 6-\mathrm{H} 62$ & 108.0 \\
\hline $\mathrm{C} 5-\mathrm{N} 4-\mathrm{H} 4$ & $107.2(12)$ & $\mathrm{N} 4-\mathrm{C} 5-\mathrm{C} 6$ & $110.00(13)$ \\
\hline $\mathrm{C} 7-\mathrm{N} 4-\mathrm{H} 4$ & $106.9(11)$ & $\mathrm{N} 4-\mathrm{C} 5-\mathrm{H} 51$ & 109.7 \\
\hline $\mathrm{C} 3-\mathrm{N} 4-\mathrm{H} 4$ & $108.6(11)$ & $\mathrm{C} 6-\mathrm{C} 5-\mathrm{H} 51$ & 109.7 \\
\hline $\mathrm{N} 4-\mathrm{C} 3-\mathrm{C} 2$ & $109.09(13)$ & $\mathrm{N} 4-\mathrm{C} 5-\mathrm{H} 52$ & 109.7 \\
\hline $\mathrm{N} 4-\mathrm{C} 3-\mathrm{H} 31$ & 109.9 & $\mathrm{C} 6-\mathrm{C} 5-\mathrm{H} 52$ & 109.7 \\
\hline $\mathrm{C} 2-\mathrm{C} 3-\mathrm{H} 31$ & 109.9 & $\mathrm{H} 51-\mathrm{C} 5-\mathrm{H} 52$ & 108.2 \\
\hline $\mathrm{N} 4-\mathrm{C} 3-\mathrm{H} 32$ & 109.9 & $\mathrm{~N} 4-\mathrm{C} 7-\mathrm{C} 8$ & $113.63(13)$ \\
\hline $\mathrm{C} 2-\mathrm{C} 3-\mathrm{H} 32$ & 109.9 & $\mathrm{~N} 4-\mathrm{C} 7-\mathrm{H} 71$ & 108.8 \\
\hline $\mathrm{H} 31-\mathrm{C} 3-\mathrm{H} 32$ & 108.3 & $\mathrm{C} 8-\mathrm{C} 7-\mathrm{H} 71$ & 108.8 \\
\hline $\mathrm{O} 1-\mathrm{C} 2-\mathrm{C} 3$ & $111.28(13)$ & N4-C7-H72 & 108.8 \\
\hline $\mathrm{O} 1-\mathrm{C} 2-\mathrm{H} 21$ & 109.4 & $\mathrm{C} 8-\mathrm{C} 7-\mathrm{H} 72$ & 108.8 \\
\hline $\mathrm{C} 3-\mathrm{C} 2-\mathrm{H} 21$ & 109.4 & $\mathrm{H} 71-\mathrm{C} 7-\mathrm{H} 72$ & 107.7 \\
\hline $\mathrm{O} 1-\mathrm{C} 2-\mathrm{H} 22$ & 109.4 & $\mathrm{C} 7-\mathrm{C} 8-\mathrm{Cl} 1$ & $114.08(12)$ \\
\hline $\mathrm{C} 3-\mathrm{C} 2-\mathrm{H} 22$ & 109.4 & $\mathrm{C} 7-\mathrm{C} 8-\mathrm{H} 81$ & 108.7 \\
\hline $\mathrm{H} 21-\mathrm{C} 2-\mathrm{H} 22$ & 108.0 & $\mathrm{C} 11-\mathrm{C} 8-\mathrm{H} 81$ & 108.7 \\
\hline $\mathrm{C} 2-\mathrm{O} 1-\mathrm{C} 6$ & $109.92(12)$ & $\mathrm{C} 7-\mathrm{C} 8-\mathrm{H} 82$ & 108.7 \\
\hline $\mathrm{O} 1-\mathrm{C} 6-\mathrm{C} 5$ & $111.36(13)$ & $\mathrm{C} 11-\mathrm{C} 8-\mathrm{H} 82$ & 108.7 \\
\hline $\mathrm{O} 1-\mathrm{C} 6-\mathrm{H} 61$ & 109.4 & $\mathrm{H} 81-\mathrm{C} 8-\mathrm{H} 82$ & 107.6 \\
\hline $\mathrm{C} 5-\mathrm{C} 6-\mathrm{H} 61$ & 109.4 & & \\
\hline $\mathrm{C} 5-\mathrm{N} 4-\mathrm{C} 3-\mathrm{C} 2$ & $56.13(16)$ & $\mathrm{C} 3-\mathrm{N} 4-\mathrm{C} 5-\mathrm{C} 6$ & $-55.60(16)$ \\
\hline $\mathrm{C} 7-\mathrm{N} 4-\mathrm{C} 3-\mathrm{C} 2$ & $-177.50(12)$ & $\mathrm{O} 1-\mathrm{C} 6-\mathrm{C} 5-\mathrm{N} 4$ & $57.83(17)$ \\
\hline $\mathrm{N} 4-\mathrm{C} 3-\mathrm{C} 2-\mathrm{O} 1$ & $-59.50(17)$ & $\mathrm{C} 5-\mathrm{N} 4-\mathrm{C} 7-\mathrm{C} 8$ & $-73.70(17)$ \\
\hline $\mathrm{C} 3-\mathrm{C} 2-\mathrm{O} 1-\mathrm{C} 6$ & $60.97(17)$ & $\mathrm{C} 3-\mathrm{N} 4-\mathrm{C} 7-\mathrm{C} 8$ & $162.71(13)$ \\
\hline $\mathrm{C} 2-\mathrm{O} 1-\mathrm{C} 6-\mathrm{C} 5$ & $-59.89(17)$ & $\mathrm{N} 4-\mathrm{C} 7-\mathrm{C} 8-\mathrm{Cl} 1$ & $86.66(15)$ \\
\hline $\mathrm{C} 7-\mathrm{N} 4-\mathrm{C} 5-\mathrm{C} 6$ & $179.93(13)$ & & \\
\hline
\end{tabular}

Hydrogen-bond geometry $\left(\AA,{ }^{\circ}\right)$

\begin{tabular}{lllll}
\hline$D-\mathrm{H} \cdots A$ & $D-\mathrm{H}$ & $\mathrm{H} \cdots A$ & $D \cdots A$ & $D-\mathrm{H} \cdots A$ \\
\hline $\mathrm{N} 4-\mathrm{H} 4 \cdots \mathrm{Cl} 2$ & $0.883(19)$ & $2.16(2)$ & $3.0435(14)$ & $178(2)$ \\
\hline
\end{tabular}

